\title{
Airway Management of Massive Hemoptysis in a Tracheostomized Patient: A Clinical Approach
}

\author{
Tariq Janjua ${ }^{1}$, Fawad Bangash², Luis R Moscote-Salazar ${ }^{3}$
}

\begin{abstract}
Airway management in a critical care environment is one of the most important and difficult procedures. This procedure is usually complicated with marked derangements in patients. These derangements lead to minimal time to act and maneuver intubation with no or minimal impact on the underlying condition. One of these conditions is the continued critical care need for tracheotomized patients. The complications of tracheostomy and airway management are challenging tasks. One of the complications is airway compromise due to hemorrhage requiring airway control. Here, we describe two cases with a proposed pathway.

Keywords: Critical care, Hemoptysis, Tracheostomy.

Panamerican Journal of Trauma, Critical Care \& Emergency Surgery (2021): 10.5005/jp-journals-10030-1320
\end{abstract}

\section{Case Descriptions}

\section{Case 1}

A 54-year-old female patient with no past medical history admitted to intensive care unit with high-grade fever, tachypnea, and low pulse oximetry. Her COVID-19 PCR came back positive and due to low PF ratio ARDS, she was placed on venovenous extracorporeal oxygenation (VV ECMO) followed by intubation. After 4 weeks of being on VV-ECMO, a formal bedside percutaneous tracheostomy (PDT) was performed with placement of size 6 cuffed Shiley ${ }^{\mathrm{TM}}$ (Medtronic, Minneapolis, USA). The patient tolerated the procedure well and over the next 48 hours weaned off the ventilator to a high-flow trach tube. Her heparin infusion for VV-ECMO continued except the day of PDT. On the 3rd day of PDT, bedside staff noticed fresh blood around the trach site which increased overnight. The patient was placed back on the ventilator. Local dressing changes did not help with hemostasis. At that stage, heparin infusion was stopped and an emergent ENT consult was obtained. The prothrombotic agent was applied around the site with no stoppage of bleeding. Meanwhile, the patient's oxygenation dropped leading to increased blood flow on VV-ECMO flow. An emergent bronchoscopic examination with full ventilator support through size 6 tracheostomy showed frank clots in the lower trachea. The patient was deeply sedated and paralyzed followed by conversion to size 6 oral endotracheal tube (ETT). Video laryngoscopy with Glidescope ${ }^{R}$ (Verathon, USA) size 4 blade showed total occlusion of the larynx with clotted blood. Suction is done to visualize vocal cords. An endotracheal size 6 tube secured. The tracheostomy got removed and the ENT surgery team started working on local hemostasis. The intensivist proceeded with bronchoscopic confirmation of airway blood clots removal which was not possible due to a smaller bronchoscope. With the help of an exchange catheter, size 6 ETT exchanged to size 8 ETT, which was pulled superior to the tracheostomy site for the ENT surgeon to continue fixing the bleeding. A therapeutic bronchoscope is used to clean the lower trachea, both main bronchi and segmental bronchi of blood clots and fresh blood. After hemostasis, a new tracheostomy was inserted with the removal of ETT. The patient was weaned off
${ }^{1}$ Cradiac Critical Care Unit, Methodist Hospital, Houston, Texas, USA

${ }^{2}$ Critical Care Department, St. Agnes Hospital, Fond Du Lac, Wisconsin, USA

${ }^{3}$ Department of Neurosurgery, Universdiad de Cartagena, Cartagena, Colombia, South America

Corresponding Author: Luis R Moscote-Salazar, Department of Neurosurgery, Universdiad de Cartagena, Cartagena, Colombia, South America, Phone: +573385978, e-mail: rafaelmoscote21@gmail.com

How to cite this article: Janjua T, Bangash F, Moscote-Salazar LR. Airway Management of Massive Hemoptysis in a Tracheostomized Patient: A Clinical Approach. Panam J Trauma Crit Care Emerg Surg 2021;10(2):85-86.

Source of support: Nil

Conflict of interest: None

paralysis and sedation over the next 24 hours and continued on heparin for VV-ECMO.

\section{Case 2}

A 75-year-old male patient with a past medical history of morbid obesity (BMI 45), diabetes mellitus 2, CHF, hypertension, CKD III, and OSA who was evaluated for severe aortic stenosis and 3 vessel $C A D$ by cardiothoracic surgery. The patient underwent aortic valve replacement and 3 vessel CABG. The postoperative course was complicated by respiratory failure and atrial fibrillation. Eventually, the patient underwent a tracheostomy and was started on warfarin for anticoagulation. On postoperative day 5 , the patient developed massive hemoptysis and received a massive transfusion and correction of INR with FFP. Local measures to control hemoptysis included emergent bronchoscopy and epinephrine instillation via tracheostomy tube were unsuccessful. At that stage, orotracheal intubation was done after the removal of the tracheostomy. In the operating room, the tracheostomy site was explored surgically leading to control of bleeding. Conversion to tracheostomy was done in the operating room and he was brought back to the critical care unit. The patient was eventually discharged on postoperative day 21 to a long-term care acute care facility. 
Flowchart 1: Flow diagram shows a clinical approach for dealing with a catastrophic hemorrhage from a tracheostomy in the intensive care unit. OT, orotracheal; OTI, orotracheal intubation; T, tracheostomy; TIF, tracheoinnominate fistula; OR, operating room; MVS, mechanical ventilator support

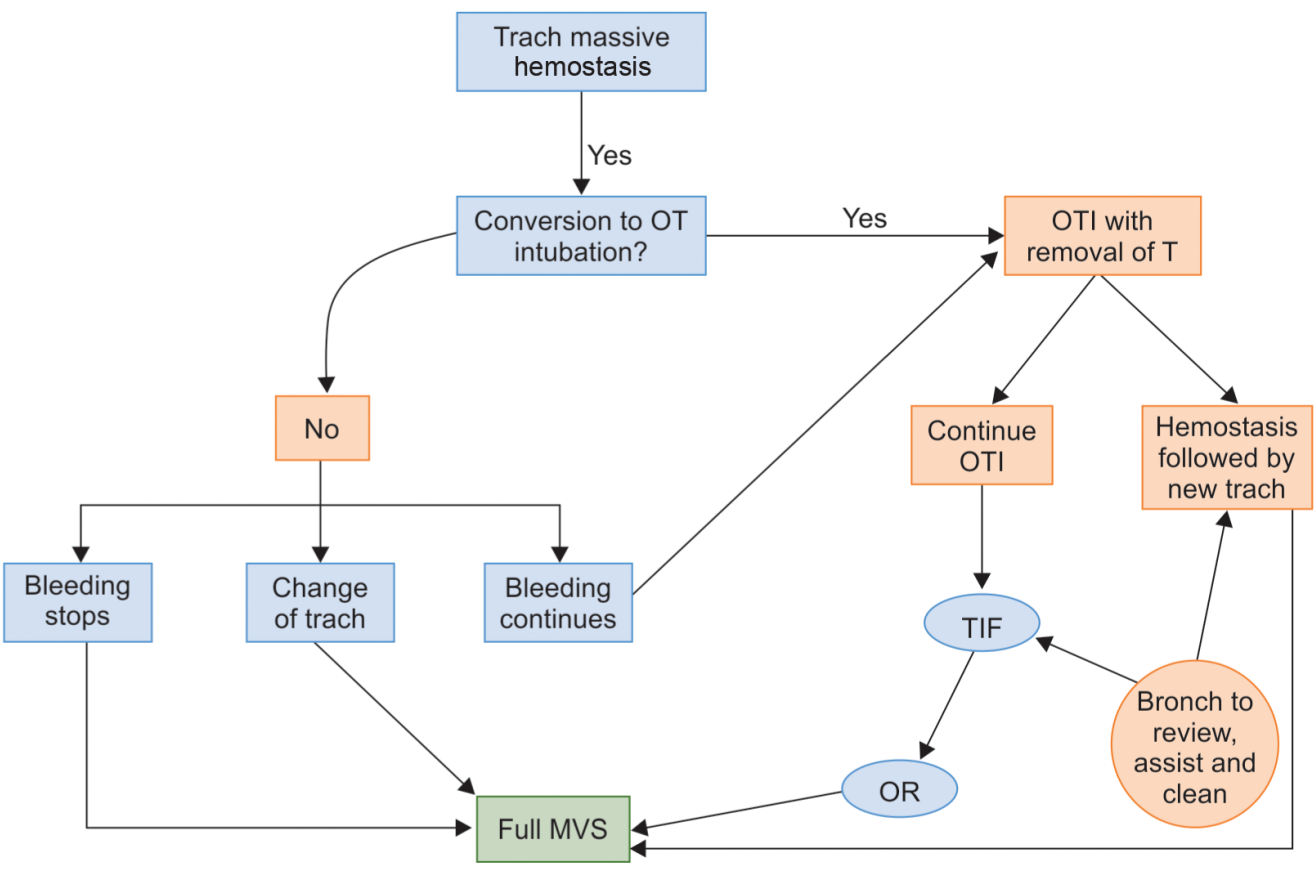

Catastrophic hemorrhage in intensive care due to tracheostomy leads to high morbidity and mortality. Early recognition and hemostasis are required. Tracheoinnominate fistula (TIF) is a life-threatening complication with mortality close to $100 \%$ if not corrected. Early bleeding is usually local and related to factors like anticoagulation while late bleeding is related to tracheostomy itself. ${ }^{1-3}$ The innominate artery crosses from left to right at the ninth tracheal ring but can be as high as the sixth ring. Factors that can lead to TIF include pressure necrosis from cuff pressure, tracheal tube tip pushing against the trachea, placement too low (below the third ring), or local tracheal wall weakness due to radiation or steroids. Local hemostasis followed by definitive corrective surgery is required. ${ }^{4,5}$ Tracheal tube pulled forward with high cuff pressure can sometimes tamponade the artery while preparation is made to proceed to the operating room. Placement of index finger behind the sternum with thumb outside can help with tamponade. At this stage, tracheostomy is removed and OTI is done.

In essence, a high vigilance is required for tracheostomy hemorrhage in the intensive care and airway control with hemostasis is the key aim. Flowchart 1 shows a pathway to manage the airway in the intensive care with catastrophic tracheostomyinduced hemorrhage.

\section{References}

1. Grant CA, Dempsey G, Harrison J, et al. Tracheo-innominate artery fistula after percutaneous tracheostomy: three case reports and a clinical review. Br J Anaesth 2006;96(1):127-131. DOI: 10.1093/bja/ aei282.

2. Gasparri MG, Nicolosi AC, Almassi GH. A novel approach to the management of tracheoinnominate artery fistula. Ann Thorac Surg 2004;77(4):1424-1426. DOI: 10.1016/S0003-4975(03)01000-2.

3. Bradley PJ. Bleeding around a tracheostomy wound: what to consider and what to do? J Laryngol Otol 2009;123(9):952-956. DOI: 10.1017/ S002221510900526X.

4. Ridley RW, Zwischenberger JB. Tracheoinnominate fistula: surgical management of an iatrogenic disaster. J Laryngol Otol 2006;120(8):676-680. DOI: 10.1017/S0022215106001514.

5. Epstein SK. Late complications of tracheostomy. Respir Care 2005;50(4):542-549. 